proposed to be done is the physical fact of penetration of her body by the male organ or, if that is not proved, (b) that the act of penetration proposed is one of sexual connexion as distinct from one of totally different character.' It is my contention that this standard of knowledge is insufficient to allow a person to protect $\mathrm{him} /$ herself against the commonly recognized consequences of sexual acts, namely pregnancy and sexually transmitted diseases. Although the literature suggests that increasing the required standard of knowledge to encompass these facts would mean that many persons with mental impairment would be deemed incapable of consent, I argue that consent that is not based on a standard of knowledge sufficient to allow an individual to safeguard their own interests cannot be considered true consent. Law reform is required so that consent to sexual acts more closely resembles the informed consent required for medical treatment.

\section{Cognition and volition: a melancholic condition}

\section{Green'1 D Hadzi-Pavlovic' ${ }^{1}$, G Malhï ${ }^{2,3,4}$}

'School of Psychiatry, University of New South Wales; ${ }^{2}$ Academic Discipline of Psychological Medicine, Northern Clinical School, The University of Sydney; ${ }^{3}$ The Black Dog Institute, Sydney; and "Prince of Wales Medical Research Institute, Sydney, Australia

Background: Melancholia, a subtype of depression described as a specifier in DSM-IV, also forms a component of bipolar disorder (BD) and is distinguished from nonmelancholic depression on the basis of additional psychomotor disturbance (PMD). The latter comprises both retardation and agitation; concomitant cognitive disturbances are clinically observable and identified by patients as 'a difficulty in thinking', with lapses in concentration and decision-making impairments.

Methods: A literature review of experimental studies of cognition in melancholia was undertaken alongside a review of literature on bradyphrenia (slowed cognition) within various neuropsychiatric conditions. Particular emphasis was assigned to studies that attempted to distinguish PMD from processing speed on cognitive tasks, in terms of both functional neuroanatomy and relationships with impaired volition. The implications for future studies of cognition in melancholia are carefully critiqued.

Results: There has been relatively little empirical investigation of cognition and volition in melancholia. Studies thus far report partitioning of the neuropsychological profile of depression across melancholic and nonmelancholic subtypes implicating distinct neural dysfunction within dorsal and ventral prefrontal networks. In comparison, research examining psychomotor retardation and impaired volition has gravitated toward abnormal functioning within basal ganglia and related networks, with the archetypal example of this being Parkinson's disease.

Conclusions: Further empirical examination of cognition in depressive subtypes is required to evaluate the potential contribution of impaired volition to psychomotor retardation and distinct neuropsychological deficits in melancholia. Evidence for a cognitive marker of melancholia may prove useful for refining diagnostic criteria and elucidating functional brain networks that subserve endogeneity.

\section{Childhood risk for obsessive compulsive disorder \\ JR Grisham', R Poulton², G Andrews'}

'University of New South Wales, Sydney, Australia; and 2Dunedin Multidisciplinary Health and Development Research Unit, Dunedin, New Zealand

Background: Identifying childhood risk factors for obsessive compulsive disorder (OCD) is essential for improved understanding of its etiology and pathogenesis. Previous research has been largely reliant on retrospective self-report measurement of risk factors. The purpose of the current study was to link early appearing temperamental traits, behaviour, psychiatric symptoms and environmental stressors to the later emergence of OCD.

Methods: Longitudinal data were collected from participants of the Dunedin Multidisciplinary Health and Developmental Study. Study members were assessed from birth to adulthood on various measures of health and behaviour. Childhood temperament, behaviour, psychiatric symptoms and psychosocial stressors were linked with adult diagnostic outcomes.

Results: Preliminary findings suggest that certain types of childhood temperament and behaviour styles were associated with increased risk of developing OCD in adulthood. Childhood symptoms of OCD and childhood abuse were also risk factors for adult OCD diagnosis. Associations between childhood factors and OCD were largely independent of gender or socioeconomic status.

Conclusions: Results of the current study suggests that within-child characteristics and adverse life events play a role in the development of OCD. Findings also underline the need for targeted interventions for children with high-risk temperament or behavioural styles or childhood OCD symptoms. 